Journal for ImmunoTherapy of Cancer

\title{
Tislelizumab in Chinese patients with advanced solid tumors: an open-label, non-comparative, phase $1 / 2$ study
}

Lin Shen, ${ }^{1}$ Jun Guo, ${ }^{1}$ Qingyuan Zhang, ${ }^{2}$ Hongming Pan, ${ }^{3}$ Ying Yuan, ${ }^{4}$ Yuxian Bai, ${ }^{2}$ Tianshu Liu, ${ }^{5}$ Qing Zhou, ${ }^{6}$ Jun Zhao, ${ }^{1}$ Yongqian Shu, ${ }^{7}$ Xiaoming Huang, ${ }^{8}$ Siyang Wang, ${ }^{9}$ Jie Wang (D) , ${ }^{10}$ Aiping Zhou, ${ }^{10}$ Dingwei Ye, ${ }^{11}$ Ting Sun, ${ }^{12}$ Yujuan Gao, ${ }^{13}$ Silu Yang, ${ }^{13}$ Zuobai Wang, ${ }^{13}$ Jian Li, ${ }^{13}$ Yi-Long Wu (i) ${ }^{6}$

To cite: Shen L, Guo J, Zhang Q, et al. Tislelizumab in Chinese patients with advanced solid tumors: an open-label, noncomparative, phase $1 / 2$ study. Journal for ImmunoTherapy of Cancer 2020;8:e000437. doi:10.1136/jitc-2019-000437

- Additional material is published online only. To view please visit the journal online (http://dx.doi.org/10.1136/jitc2019-000437).

LS and JG are joint first authors.

Accepted 03 March 2020
Check for updates

(C) Author(s) (or their employer(s)) 2020. Re-use permitted under CC BY-NC. No commercial re-use. See rights and permissions. Published by BMJ.

For numbered affiliations see end of article.

Correspondence to

Dr Yi-Long Wu; syylwu@live.cn

\section{ABSTRACT}

Background Tislelizumab is an investigational, humanized, IgG4 monoclonal antibody with high affinity and binding specificity for programmed cell death-1 (PD1) that was engineered to minimize binding to Fc $\gamma R$ on macrophages in order to abrogate antibody-dependent phagocytosis, a mechanism of T-cell clearance and potential resistance to anti-PD-1 therapy.

Methods The purpose of this phase 1/2, open-label, non-comparative study was to examine the safety, tolerability, and antitumor activity of tislelizumab in adult ( $\geq 18$ years) Chinese patients with histologically or cytologically confirmed advanced solid tumors with measurable disease. The phase 1 portion of the study consisted of a dose-verification study and a pharmacokinetic (PK) substudy; phase 2 was an indication-expansion study including 11 solid tumor cohorts. Patients previously treated with therapies targeting PD-1 or its ligand, programmed cell death ligand-1 were excluded. During dose-verification, doselimiting toxicities (DLTs) were monitored; safety and tolerability were examined and the previously determined recommended phase 2 dose (RP2D) was verified. The primary endpoint of phase 2 was investigator-assessed objective response rate per Response Evaluation Criteria in Solid Tumors V.1.1.

Results As of December 1, 2018, 300 patients were treated with tislelizumab $200 \mathrm{mg}$ intravenously once every 3 weeks (Q3W). Median duration of follow-up was 8.1 months (range 0.2-21.9). No DLTs were reported during the phase 1 dose-verification study and the RP2D was confirmed to be $200 \mathrm{mg}$ intravenously Q3W. Most treatment-related adverse events (62\%) were grade 1 or 2 , with the most common being anemia $(n=70 ; 23 \%)$ and increased aspartate aminotransferase $(n=67 ; 22 \%)$. of the 251 efficacy evaluable patients, 45 (18\%) achieved a confirmed clinical response, including one patient from the PK substudy who achieved a complete response. Median duration of response was not reached for all except the nasopharyngeal carcinoma cohort (8.3 months). Antitumor responses were observed in multiple tumor types.

Conclusions Tislelizumab was generally well tolerated among Chinese patients. Antitumor activity was observed in patients with multiple solid tumors.

Trial registration number CTR20160872.

\section{BACKGROUND}

Cancer is the second leading cause of death worldwide $^{1}$; in 2018, there were an estimated 18.1 million new cancer cases and 9.6 million cancer-related deaths. ${ }^{2}$ With the world's largest population, about one-fifth of cancer cases occur in China. ${ }^{3}$ Despite improvements in overall survival among patients with cancer over the last decade in China, survival remains lower than in many other developed countries. ${ }^{4}$ Therefore, there is an unmet medical need for more novel, effective, and safe therapies to be made available to Chinese patients with cancer, especially for the treatment of tumors that have shown distinctive clinical features and/or pathology among Chinese or East Asian patients, such as non-small cell lung cancer (NSCLC), hepatocellular carcinoma (HCC), gastric cancer (GC), nasopharyngeal carcinoma (NPG), esophageal squamous cell carcinoma (ESCC), and melanoma.

One mechanism by which tumor cells escape immune surveillance is through changes in the expression of specific receptors and ligands involved in the immune checkpoint pathway. Programmed cell death-1 (PD-1) is a cell surface receptor that is expressed on activated $\mathrm{T}$ cells as part of the adaptive immune response and which inhibits T-cell signaling when it binds to its ligands, PD-L1 and PD-L2. ${ }^{5}$ Both PD-L1 and PD-L2 are often overexpressed by tumor cells to evade immune surveillance, detection, and eventual destruction. ${ }^{6-12}$ Antibodies against PD-1 block the binding of PD-L1 or PD-L2 to PD-1, counteracting checkpoint-mediated T-cell suppression and permitting $\mathrm{T}$ cells to induce tumor cell death. ${ }^{13}{ }^{14}$ In clinical trials, monoclonal antibodies against the immune checkpoint inhibitory receptor PD-1 have demonstrated objective responses in patients with multiple malignancies. ${ }^{15}$ Antibodies 
targeting PD-1/PD-L1 have been approved for multiple tumor types by the US Food and Drug Administration (FDA) including several that are the focus of the clinical trial described in this article (melanoma, NSCLC, GC, renal cell carcinoma [RCC], urothelial carcinoma [UC], microsatellite instability-high [MSI-H]/deficient mismatch repair [dMMR] cancer, and hepatocellular carcinoma $[\mathrm{HCC}]$ ).

Tislelizumab is an investigational, humanized, IgG4 monoclonal antibody with high affinity and binding specificity for PD-1 that was engineered to minimize binding to Fc $\gamma$ Rs on macrophages in order to abrogate antibody-dependent cellular phagocytosis, a mechanism of T-cell clearance and potential resistance to anti-PD-1 therapy. ${ }^{16} 17$ Tislelizumab shows higher affinity to PD-1 when compared with pembrolizumab and nivolumab, with an 100-fold slower off-rate than pembrolizumab and $\sim 50$-fold slower off-rate than nivolumab. ${ }^{18}$ These differences in binding affinity can be partially attributed to tislelizumab's different binding orientation to PD-1 when compared with pembrolizumab and nivolumab; the binding surface on PD-1 for tislelizumab partially overlaps with that for pembrolizumab, but differs significantly from that for nivolumab. ${ }^{18}$ The safety of tislelizumab was initially evaluated in a first-in-human phase 1A/B study (NCT02407990), which showed that singleagent tislelizumab was generally well tolerated. ${ }^{19} 20$ The pharmacokinetics (PK) of tislelizumab were linear and systemic clearance was not affected by body weight, which supported fixed dosing. ${ }^{19}$ Furthermore, because no unexpected treatment-related adverse events (TRAEs) occurred in the $200 \mathrm{mg}$ fixed-dose cohort, and the observed serum exposure of a $200 \mathrm{mg}$ dose fell between serum exposures observed after $2 \mathrm{mg} / \mathrm{kg}$ and $5 \mathrm{mg} / \mathrm{kg}$ doses, tislelizumab $200 \mathrm{mg}$ intravenously Q3W was the recommended dose for subsequent pivotal studies.

This phase $1 / 2$ study sought to verify the recommended phase 2 dose (R2PD) determined in a previous first-inhuman study, ${ }^{20}$ and examined the safety, tolerability, and antitumor activity of tislelizumab among Chinese patients with advanced solid tumors.

\section{METHODS}

\section{Study design and patients}

This ongoing, multicenter, open-label, phase 1/2 study (CTR20160872) conducted in 16 centers in China was initiated on December 28, 2016. Phase 1 included a doseverification study, as well as a PK substudy for the purpose of comparing two different antibody manufacturing processes and scales (500L-Final Manufacturing Process (FMP) and 2000L-FMP); phase 2 was an indication expansion across 11 cohorts of various tumor types (online supplementary figure S1). Data from the PK substudy were pooled for efficacy and safety analyses included in this article, but PK results are not reported herein.

Patients aged $\geq 18$ years with histologically or cytologically confirmed advanced or metastatic solid tumors with measurable disease (defined by Response Evaluation Criteria in Solid Tumors version 1.1 [RECIST V.1.1]), ${ }^{21}$ and an Eastern Cooperative Oncology Group (ECOG) performance score of $\leq 1$, were eligible for enrollment. Patients must have progressed since their last standard antitumor treatment, had no available (or refused) standard treatment, or become intolerant to treatment and have adequate organ function. Patients provided archival tumor tissues or newly obtained tumor tissue and relevant pathological reports. Key exclusion criteria included a history of severe hypersensitivity reactions to other monoclonal antibodies, prior active malignancy within 2 years of enrollment (except tumors investigated as part of this trial), prior therapies targeting PD-1 or PD-L1, and symptomatic brain metastases.

This study was performed in accordance with the ethical principles of the Declaration of Helsinki, Good Clinical Practice guidelines, and the principles of informed consent. Written informed consent was obtained from each patient prior to screening. The protocol was approved by an independent ethics committee prior to initiation.

\section{Procedures}

During dose verification, patients were treated with tislelizumab $200 \mathrm{mg}$ intravenously Q3W and initial analyses examined three to six patients who had received at least $80 \%$ of the dose and completed all safety assessments required in cycle 1 for predefined dose-limiting toxicities (DLTs) within 21 days of initial treatment. From this analysis, a safety monitoring committee determined that no DLTs were identified. The dose-verification cohort was then expanded to 20 patients for further assessment of safety, tolerability, and PK analysis. During the parallel PK substudy, 57 patients received multiple doses of tislelizumab to analyze the PK and safety of tislelizumab from two different manufacturing processes and scales. During indication expansion, patients received the RP2D. Throughout the study, safety data were regularly reviewed by a contract research organization, medical monitors, and pharmacovigilance physicians.

PD-L1 expression on tumor cells was prospectively confirmed by the central laboratory in patients with NSCLC during phase 2 using the VENTANA PD-L1 (SP263) assay ${ }^{22}$; PD-L1 positivity was defined as $\geq 10 \%$ of tumor cells with PD-L1 membrane staining at any intensity. For other enrolled patients, PD-L1 expression was retrospectively assessed.

Tislelizumab $200 \mathrm{mg}$ intravenously was administered Q3W throughout the study until patients had no evidence of continued clinical benefit, or until unacceptable toxicity, or withdrawal of consent. Safety and efficacy among patients were closely monitored throughout the study. Adverse events (AEs) were graded according to the National Cancer Institute Common Toxicity Criteria for Adverse Events (NCICTCAE) V.4.03. Tumor responses were assessed by investigators based on RECIST V.1.1, and radiological assessments of tumor response status were performed within 28 days prior to enrollment, approximately every 9 weeks in the 
first year, and every 12 weeks thereafter. Treatment beyond progression in clinically benefited patients was permitted.

\section{Outcomes}

In the dose-verification portion of phase 1 , the primary endpoints were the safety and tolerability of tislelizumab, as assessed per NCI-CTCAE V.4.03, and RP2D based on safety, tolerability, PK, and preliminary efficacy. As the mechanism of action of tislelizumab involves immune regulation, particular attention was given to immune-related AEs (irAEs). The primary endpoint of phase 2 was investigatorassessed objective response rate (ORR) per RECIST V.1.1. Secondary endpoints in phase 1 included antitumor activity and immunogenic responses; secondary endpoints of phase 2 included progression-free survival (PFS), overall survival (OS), duration of response (DoR), disease control rate (DCR), clinical benefit rate (CBR), immunogenic responses, PK, and incidence and severity of AEs. Exploratory endpoints included examination of potential predictive biomarkers including PD-L1 expression and tumor mutation status.

\section{Statistical analyses}

The sample size in the dose-verification stage of phase 1 (expected enrollment was approximately 20 patients) was determined based on the number of dose levels to be assessed and the occurrence of DLTs in each cohort. In the phase 1 PK substudy, a total of 48 patients (24 per arm) were planned to be enrolled to receive tislelizumab from two manufacturing processes and scales. Approximately 220 patients were expected to be enrolled in the indication-expansion stage of phase 2 to perform the preliminary efficacy analysis for tislelizumab monotherapy.

Categorical variables were summarized by number (percentage) of patients. Continuous variables were reported by descriptive statistics. The Clopper-Pearson method was used to estimate 95\% CIs for response rates and the Kaplan-Meier method was used to estimate medians and 95\% CIs for time-to-event variables. All patients who received tislelizumab were included in the safety analysis set. The efficacy analysis set included patients who received tislelizumab and had measurable disease per investigator according to RECIST V.1.1 at baseline and at least one postbaseline assessment. The safety results from all patients and each study phase are presented. All eligible patients for each indication that included more than 15 patients during phases 1 and 2 were pooled for analysis by indication.

\section{RESULTS}

\section{Patient disposition, demographics, and baseline disease characteristics}

As of December 1, 2018, 386 patients were screened and 300 were treated with at least one dose of tislelizumab. As shown in table 1, 20 patients were enrolled in the doseverification study, 57 in the PK substudy, and 223 in phase 2 of this trial. The median duration of follow-up for all patients was 8.1 months (range 0.2-21.9). A total of 223 patients had discontinued treatment, the most common reason being disease progression $(\mathrm{n}=159 ; 71.3 \%) ; 77$ patients remained on treatment.

Most patients treated were male $(n=207 ; 69 \%)$ and had an ECOG performance status of $1(n=220 ; 73 \%)$. Of 288 patients $(96 \%)$ who received prior anticancer drug therapy, the majority had two or more prior regimens of antitumor treatment $(\mathrm{n}=204,71 \%)$, with $23 \%$ of patients receiving four or more prior regimens. Across the study, the median time from initial diagnosis to study entry was 19.9 months (range 0.4-189.5).

\section{Safety and tolerability profile}

Across the study, the median number of on-study treatment cycles was six (range 1-32), with a median duration of treatment of 18 weeks (range 0.9-95.7). During the dose-verification portion of the trial, the RP2D was confirmed to be tislelizumab $200 \mathrm{mg}$ intravenously Q3W. No DLTs were reported during dose verification. Across the study, the most common treatment-emergent AEs (TEAEs) reported were anemia $(\mathrm{n}=104 ; 35 \%)$, increased aspartate aminotransferase $(\mathrm{n}=75 ; 25 \%)$, and increased alanine aminotransferase $(\mathrm{n}=67 ; 22 \%)$ (online supplementary table S2). Grade $\geq 3$ TEAEs were reported in $119(40 \%)$ patients, most commonly anemia $(\mathrm{n}=17$; $6 \%)$, increased gamma-glutamyl transferase $(\mathrm{n}=14 ; 5 \%)$, and increased aspartate aminotransferase $(\mathrm{n}=10 ; 3 \%)$. TEAEs led to discontinuation in $25(8 \%)$ patients; the only TEAEs that led to discontinuation in more than one patient were autoimmune myocarditis, lung infection, central nervous system metastases, and death ( $\mathrm{n}=2 \mathrm{each}$ ). Most AEs considered related to tislelizumab by the investigator were of mild or moderate severity, with the most common being anemia $(n=70 ; 23 \%)$, increased aspartate aminotransferase $(n=67 ; 22 \%)$, and increased alanine aminotransferase $(n=59 ; 20 \%)$. Grade $\geq 3$ TRAEs were reported in 99 patients (33\%), with the most common being increased gamma-glutamyl transferase $(n=12 ; 4 \%)$, anemia $(\mathrm{n}=9 ; 3 \%)$, and increased aspartate aminotransferase $(n=8 ; 3 \%)$ (table 2$)$.

Seventy-six patients (25\%) developed serious AEs including pneumonia $(\mathrm{n}=7)$, lung infection $(\mathrm{n}=4)$, intestinal obstruction $(n=4)$, and upper gastrointestinal hemorrhage $(n=4)$. A total of 145 deaths occurred during the study, $124(86 \%)$ of which were due to disease progression, $12(9 \%)$ due to AEs, 8 (6\%) due to unknown causes, and one due to drug-induced liver injury. Of 12 patients experiencing a TEAE resulting in a fatal outcome, nine were considered possibly unrelated to study drug; an additional two were considered not related. One patient had an $\mathrm{AE}$ (brain edema) considered by investigators to be possibly related to tislelizumab. This 62 -year-old male patient entered phase 2 of the study with GC that had metastasized to the brain, liver, and lymph nodes; his baseline MRI indicated multiple intracranial metastases with surrounding edema. On study day 25, the patient died due to brain edema following significant progression of 
Table 1 Demographic and baseline characteristics

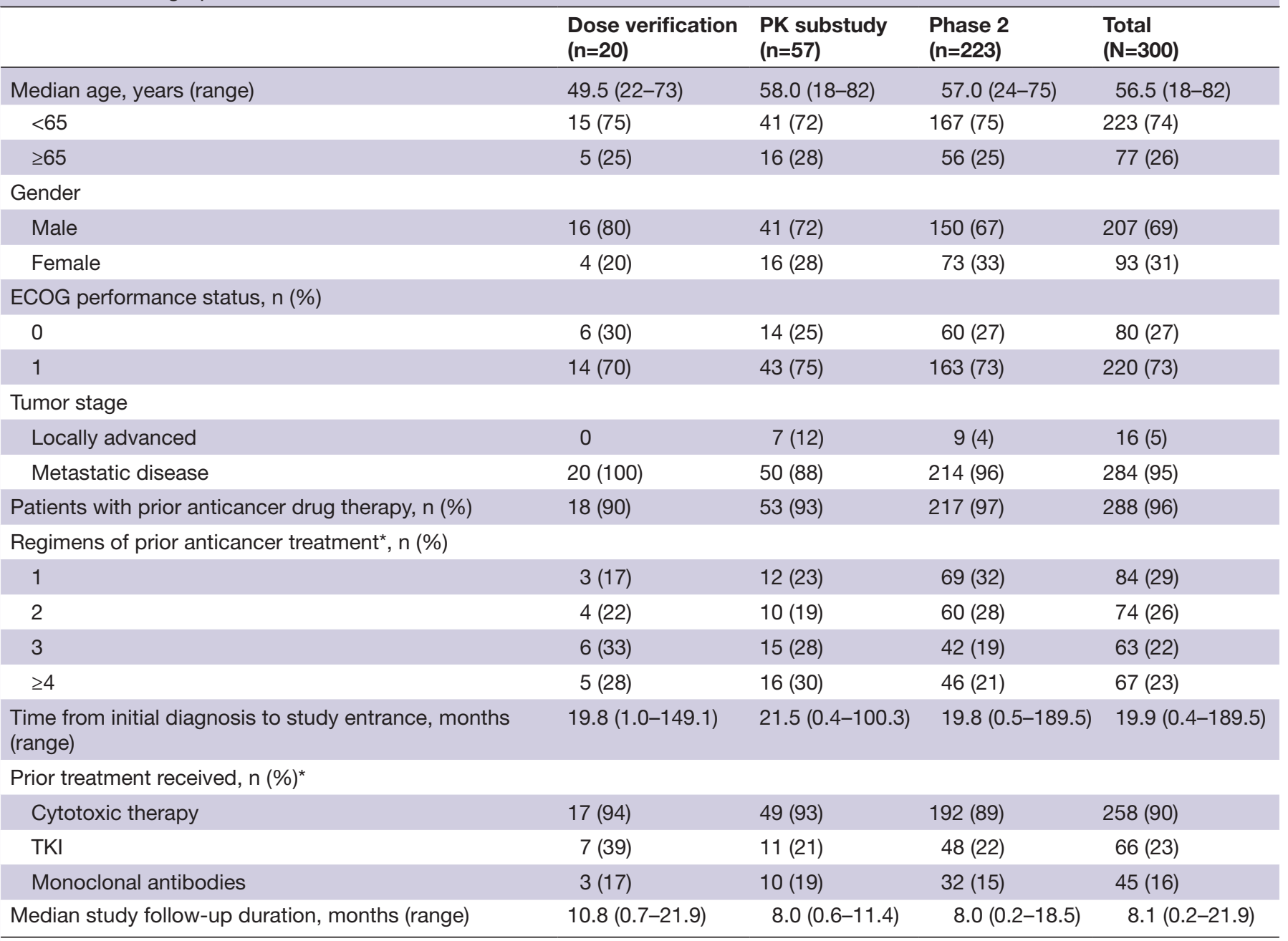

*Percentage based on patients receiving prior therapies.

ECOG, Eastern Cooperative Oncology Group; PK, pharmacokinetic; TKI, tyrosine kinase inhibitor.

brain metastases after receiving one dose of study treatment and had evidence of disease progression in the brain.

Due to endogenous function and mechanism of action, inhibition of checkpoint proteins involved in the PD-1/ PD-L1 pathway can be associated with irAEs, which most often occur in skin and gastrointestinal systems. ${ }^{23}$ These irAEs, reported in 171 patients $(57 \%)$ in the current study, could potentially limit the benefit of immunotherapy. Among all patients, the most common irAEs were increased aspartate aminotransferase $(n=59 ; 20 \%)$ and increased alanine aminotransferase $(\mathrm{n}=54 ; 18 \%)$. Thirty-three patients (11\%) experienced at least one irAE of grade $\geq 3$, the most common of which were increased gamma-glutamyl transferase $(\mathrm{n}=12 ; 4 \%)$ and increased aspartate aminotransferase $(\mathrm{n}=8 ; 3 \%)$ (table 3$)$.

\section{Preliminary antitumor activity}

Of 251 efficacy-evaluable patients from the entire study, 45 (18\%) patients achieved a confirmed response, including one patient with larynx squamous cell carcinoma from the
PK substudy who underwent surgery and two regimens of chemoradiotherapy/chemotherapy before study entry and achieved a complete response (CR). Responses were observed in all indications with more than 15 patients enrolled across the study. Indications with an ORR $\geq 15 \%$ included NPC (43\%), MSI-H/dMMR solid tumors (19\%), NSCLC (18\%), GC (17\%), HCC (17\%), and melanoma $(15 \%)$ (table 4). As of December 1, 2018, the median DoR was not mature for all indications, except for the NPC cohort, which had a DoR of 8.3 months (range 3.9 to not estimable) with a median follow-up of 4.8 months (figure 1). CBR was defined as the rate of patients who achieved confirmed CR, partial response (PR), or stable disease (SD) with a duration $\geq 16$ weeks. Indications with a CBR $\geq 50 \%$ included NPC (81\%), NSCLC (54\%), RCC (52\%), MSI-H/dMMR solid tumors $(50 \%)$, and HCC (50\%); all other indications had CBRs $\geq 25 \%$. Responses to tislelizumab were seen in multiple tumor types regardless of PD-L1 expression (table 5). 
Table 2 Treatment-related adverse events in $\geq 5 \%$ of patients (safety analysis set)

\begin{tabular}{|c|c|c|c|c|c|c|c|c|}
\hline \multirow[b]{2}{*}{ Patients with an AE, n (\%) } & \multicolumn{2}{|c|}{$\begin{array}{l}\text { Dose verification } \\
(n=20)\end{array}$} & \multicolumn{2}{|c|}{$\begin{array}{l}\text { PK substudy } \\
(n=57)\end{array}$} & \multicolumn{2}{|l|}{$\begin{array}{l}\text { Phase } 2 \\
(n=223)\end{array}$} & \multicolumn{2}{|l|}{$\begin{array}{l}\text { Total } \\
(\mathrm{N}=300)\end{array}$} \\
\hline & $\begin{array}{l}\text { Grade } \\
1-2\end{array}$ & Grade $\geq 3$ & $\begin{array}{l}\text { Grade } \\
1-2\end{array}$ & Grade $\geq 3$ & $\begin{array}{l}\text { Grade } \\
1-2\end{array}$ & Grade $\geq 3$ & $\begin{array}{l}\text { Grade } \\
1-2\end{array}$ & Grade $\geq 3$ \\
\hline Anemia & $8(40)$ & 0 & $11(19)$ & $2(4)$ & $42(19)$ & $7(3)$ & $61(20)$ & $9(3)$ \\
\hline $\begin{array}{l}\text { Aspartate aminotransferase } \\
\text { increased }\end{array}$ & $6(30)$ & 0 & $10(18)$ & $1(2)$ & $43(19)$ & $7(3)$ & $59(20)$ & $8(3)$ \\
\hline Proteinuria & $7(35)$ & 0 & $8(14)$ & 0 & $27(12)$ & $1(<1)$ & $42(14)$ & $1(<1)$ \\
\hline Blood bilirubin increased & $9(45)$ & 0 & $5(9)$ & 0 & $26(12)$ & 0 & $40(13)$ & 0 \\
\hline Hypothyroidism & $3(15)$ & 0 & $11(19)$ & 0 & $19(9)$ & 0 & $33(11)$ & 0 \\
\hline White cell count decreased & $5(25)$ & $1(5)$ & $1(2)$ & 0 & $25(11)$ & $1(<1)$ & $31(10)$ & $2(<1)$ \\
\hline Decreased appetite & $1(5)$ & 0 & $5(9)$ & 0 & $15(7)$ & $2(<1)$ & $21(7)$ & $2(1)$ \\
\hline Malaise & 0 & 0 & $2(4)$ & $1(2)$ & $17(8)$ & $1(<1)$ & $19(6)$ & $2(<1)$ \\
\hline Rash & $1(5)$ & 0 & $4(7)$ & 0 & $14(6)$ & $1(<1)$ & $19(6)$ & $1(<1)$ \\
\hline Weight decreased & $1(5)$ & 0 & $8(14)$ & 0 & $10(4)$ & $1(<1)$ & $19(6)$ & $1(<1)$ \\
\hline Platelet count decreased & $2(10)$ & 0 & $4(7)$ & 0 & $12(5)$ & $2(<1)$ & $18(6)$ & $2(<1)$ \\
\hline Neutrophil count decreased & $2(10)$ & $2(10)$ & $1(2)$ & $1(2)$ & $12(5)$ & $2(<1)$ & $15(5)$ & $5(2)$ \\
\hline Fatigue & $3(15)$ & 0 & $2(4)$ & 0 & $13(6)$ & 0 & $18(6)$ & 0 \\
\hline Vomiting & $3(15)$ & 0 & $2(4)$ & 0 & $9(4)$ & $1(<1)$ & $14(5)$ & $1(<1)$ \\
\hline Hyperuricemia & 0 & 0 & $4(7)$ & 0 & $8(4)$ & $3(1)$ & $12(4)$ & $3(1)$ \\
\hline
\end{tabular}

$\mathrm{AE}$, adverse event; PK, pharmacokinetic.

Across all patients in the study $(n=300)$, median OS was 11.5 months (95\% CI 9.1 to 15.0$)$ with a median follow-up of 12.2 months (figure 1A). Median OS was 11.3 months $(95 \%$ CI 6.8 to 18.0$)$ in patients with melanoma, 4.8 months $(95 \%$ CI 3.6 to 8.4 ) in patients with ESCC, 4.7 months (95\% CI 2.4 to not estimable) in patients with GC, and 4.3 months in patients with urothelial bladder cancer (UC) $(95 \%$ CI 2.1 to not estimable); OS data remained immature for all other indications (table 4). Median PFS for all patients was 2.6 months (95\% CI 2.2 to 4.0 ; figure $1 \mathrm{~B}$ ) and indications with a median PFS of more than 4 months include NPC (10.4 months), MSI-H/dMMR solid tumors (6.1 months), RCC (4.1 months), HCC (4.0 months), and NSCLC (4.0 months). Among patients in the trial who had responses, most experienced durable decreases of tumor burden. These durable responses were observed in all indications, even in patients who were heavily pretreated (figure 2).

\section{DISCUSSION}

A key objective of this study was to confirm the dose regimen for Chinese patients established in a previous first-in-human study (NCT02407990). This phase 1/2 study exclusively enrolled Chinese patients $(n=300)$ with the goal of examining the safety, tolerability, and antitumor activity of tislelizumab in patients with advanced solid tumors.

Tislelizumab was generally well tolerated and no unexpected safety issues were observed in this study. Reported AEs were consistent with the overall safety profile of tislelizumab observed in previous studies, ${ }^{19} 20$ as well as those 
Table 3 Immune-related adverse events in $\geq 2 \%$ of patients (safety analysis set)

\begin{tabular}{|c|c|c|c|c|c|c|c|c|}
\hline \multirow[b]{2}{*}{$\begin{array}{l}\text { Patients with an } \\
\text { irAE, } n(\%)\end{array}$} & \multicolumn{2}{|c|}{$\begin{array}{l}\text { Dose verification } \\
(n=20)\end{array}$} & \multicolumn{2}{|c|}{$\begin{array}{l}\text { PK substudy } \\
(\mathrm{n}=57)\end{array}$} & \multicolumn{2}{|l|}{$\begin{array}{l}\text { Phase } 2 \\
(n=223)\end{array}$} & \multicolumn{2}{|l|}{$\begin{array}{l}\text { Total } \\
(\mathrm{N}=300)\end{array}$} \\
\hline & $\begin{array}{l}\text { Grade } \\
1-2\end{array}$ & Grade $\geq 3$ & Grade 1-2 & Grade $\geq 3$ & Grade 1-2 & Grade $\geq 3$ & Grade 1-2 & Grade $\geq 3$ \\
\hline $\begin{array}{l}\text { Aspartate } \\
\text { aminotransferase } \\
\text { increased }\end{array}$ & $6(30)$ & 0 & $10(18)$ & $1(2)$ & $35(16)$ & $7(3)$ & $51(17)$ & $8(3)$ \\
\hline $\begin{array}{l}\text { Alanine } \\
\text { aminotransferase } \\
\text { increased }\end{array}$ & $8(40)$ & 0 & $7(12)$ & $1(2)$ & $35(16)$ & $3(1)$ & $50(17)$ & $4(1)$ \\
\hline $\begin{array}{l}\text { Blood bilirubin } \\
\text { increased }\end{array}$ & $9(45)$ & 0 & $4(7)$ & 0 & $21(9)$ & 0 & $34(11)$ & 0 \\
\hline Hypothyroidism & $3(15)$ & 0 & $10(18)$ & 0 & $18(8)$ & 0 & $31(10)$ & 0 \\
\hline $\begin{array}{l}\text { Bilirubin conjugated } \\
\text { increased }\end{array}$ & $7(35)$ & $1(5)$ & $5(9)$ & 0 & $16(7)$ & $1(<1)$ & $28(9)$ & $2(<1)$ \\
\hline $\begin{array}{l}\text { Gamma-glutamyl } \\
\text { transferase increased }\end{array}$ & 0 & 0 & $1(2)$ & $1(2)$ & $12(3)$ & $11(5)$ & $13(4)$ & $12(4)$ \\
\hline Rash & $1(5)$ & 0 & $4(7)$ & 0 & $11(5)$ & $1(<1)$ & $16(5)$ & $1(<1)$ \\
\hline $\begin{array}{l}\text { Blood creatine } \\
\text { phosphokinase } \\
\text { increased }\end{array}$ & $2(10)$ & 0 & $2(4)$ & 0 & $8(4)$ & $1(<1)$ & $12(4)$ & $1(<1)$ \\
\hline Diarrhea & $3(15)$ & 0 & $3(5)$ & 0 & $6(3)$ & $1(<1)$ & $12(4)$ & $1(<1)$ \\
\hline Hyperthyroidism & $2(10)$ & 0 & $1(2)$ & 0 & $8(4)$ & 0 & $11(4)$ & 0 \\
\hline Pruritus & $1(5)$ & 0 & $2(4)$ & 0 & $6(3)$ & 0 & $9(3)$ & 0 \\
\hline $\begin{array}{l}\text { Blood creatine } \\
\text { increased }\end{array}$ & $1(5)$ & 0 & 0 & 0 & $6(3)$ & 0 & $7(2)$ & 0 \\
\hline Hyperglycemia & 0 & 0 & $1(2)$ & 0 & $4(2)$ & $1(<1)$ & $5(2)$ & $1(<1)$ \\
\hline Pneumonia & 0 & 0 & 0 & $2(4)$ & $2(1)$ & $2(<1)$ & $2(<1)$ & $4(1)$ \\
\hline Pruritus generalized & $1(5)$ & 0 & $3(5)$ & 0 & $2(1)$ & 0 & $6(2)$ & 0 \\
\hline
\end{tabular}

irAE, immune-related adverse event; PK, pharmacokinetic.

found in other anti-PD-1 monoclonal antibody therapy agents. ${ }^{24-27}$ The majority of AEs considered related to tislelizumab by the investigator were generally of grade $\leq 2$ severity and there were no new immune-related safety signals observed. No DLTs were reported during phase 1, and tislelizumab $200 \mathrm{mg}$ intravenously Q3W was confirmed as the RP2D in Chinese patients with advanced solid tumors.

Tislelizumab demonstrated preliminary antitumor activity across multiple tumor types. In patients with GC, ESCC, NPC, and NSCLC, objective responses were observed irrespective of PD-L1 tumor expression and those in GC and NPC were numerically higher in PD-L1positive tumors compared with PD-L1-negative tumors (table 5). In patients with HCC, melanoma, RCC, UC, and MSI-H, responses were only observed in patients with PD-L1-negative tumors under the same cut-off, but conclusions should be approached with caution due to small sample size per each indication cohort (table 5). The predictive value of PD-L1 and the optimal cut-offs per indication will be explored in both ongoing and future large, randomized clinical trials of tislelizumab.
Tislelizumab demonstrated partial responses and disease stabilization in multiple tumor types and in heavily pretreated patients. Almost all patients received at least one prior anticancer drug therapy and the majority of patients $(68 \%)$ had two or more prior regimens, with roughly a quarter of patients receiving $\geq 4$ prior regimens. Across the entire study, the overall ORR was $18 \%$ and the majority of responses were durable. Median DoR was not estimable for all except the NPC cohort (8.3 months).

Previous studies have documented differences in disease biology, incidence, and clinical outcome between patients in Western Europe/North America and China in many malignancies, including NPC, NSCLC, GC, ESCC, and melanoma. ${ }^{28-30}$ The underlying mechanisms are not conclusive but may be attributed to regional differences in early detection, living and eating habits, divergent tumor characteristics, or standard treatment regimens.

NPC is characterized by a distinct geographical distribution and is particularly prevalent in East and Southeast Asia. ${ }^{31}$ Three pathological subtypes of NPC have been identified by the WHO: keratinizing squamous, non-keratinizing, and basaloid squamous. ${ }^{31}$ The 
Table 4 Summary of efficacy by indication (safety analysis set)

\begin{tabular}{|c|c|c|c|c|c|c|c|c|c|}
\hline $\begin{array}{l}\text { Response category, } \\
\text { n (\%) }\end{array}$ & $\begin{array}{l}\text { NSCLC } \\
(n=56)\end{array}$ & $\begin{array}{l}\text { Melanoma } \\
(n=34)\end{array}$ & $\begin{array}{l}\text { ESCC } \\
(n=26)\end{array}$ & $\begin{array}{l}G C \\
(n=24)\end{array}$ & $\begin{array}{l}U C \\
(n=22)\end{array}$ & $\begin{array}{l}\mathrm{RCC} \\
(\mathrm{n}=21)\end{array}$ & $\begin{array}{l}\text { NPC } \\
(n=21)\end{array}$ & $\begin{array}{l}\mathrm{HCC} \\
(\mathrm{n}=18)\end{array}$ & $\begin{array}{l}\text { MSI-H/ } \\
\text { dMMR }^{\star} \\
(n=16)\end{array}$ \\
\hline \multicolumn{10}{|l|}{ BOR† } \\
\hline PR & $10(18)$ & $5(15)$ & $2(8)$ & $4(17)$ & $3(14)$ & $2(10)$ & $9(43)$ & $3(17)$ & $3(19)$ \\
\hline SD & $21(38)$ & $8(24)$ & $7(27)$ & $3(13)$ & $6(27)$ & $9(43)$ & $9(43)$ & $7(39)$ & $5(31)$ \\
\hline Unknown & $4(7)$ & $4(12)$ & $4(15)$ & $8(33)$ & $8(36)$ & $3(14)$ & 0 & 0 & $2(13)$ \\
\hline $\begin{array}{l}\text { ORR, confirmed } \\
95 \% \mathrm{Cl}\end{array}$ & $\begin{array}{l}18 \\
8.9 \text { to } 30.4\end{array}$ & $\begin{array}{l}15 \\
5.0 \text { to } 31.1\end{array}$ & $\begin{array}{l}8 \\
0.9 \text { to } 25.1\end{array}$ & $\begin{array}{l}17 \\
4.7 \text { to } 37.4\end{array}$ & $\begin{array}{l}14 \\
2.9 \text { to } 34.9\end{array}$ & $\begin{array}{l}10 \\
1.2 \text { to } 30.4\end{array}$ & $\begin{array}{l}43 \\
21.8 \text { to } 66.0\end{array}$ & $\begin{array}{l}17 \\
3.6 \text { to } 41.4\end{array}$ & $\begin{array}{l}19 \\
4.0 \text { to } 45.6\end{array}$ \\
\hline $\begin{array}{l}\text { Median overall } \\
\text { survival, months, } \\
95 \% \mathrm{Cl}\end{array}$ & $\begin{array}{l}\mathrm{NE} \\
10.1 \text { to } \mathrm{NE}\end{array}$ & $\begin{array}{l}11.3 \\
6.8 \text { to } 18.0\end{array}$ & $\begin{array}{l}4.8 \\
3.6 \text { to } 8.4\end{array}$ & $\begin{array}{l}4.7 \\
2.4 \text { to } \mathrm{NE}\end{array}$ & $\begin{array}{l}4.3 \\
2.1 \text { to } \mathrm{NE}\end{array}$ & $\begin{array}{l}\mathrm{NE} \\
8.0 \text { to } \mathrm{NE}\end{array}$ & $\begin{array}{l}\mathrm{NE} \\
9.2 \text { to } \mathrm{NE}\end{array}$ & $\begin{array}{l}\mathrm{NE} \\
5.5 \text { to } \mathrm{NE}\end{array}$ & $\begin{array}{l}\mathrm{NE} \\
4.2 \text { to } \mathrm{NE}\end{array}$ \\
\hline CBR $\neq, 95 \% \mathrm{Cl}$ & $\begin{array}{l}52 \\
38.0 \text { to } 65.3\end{array}$ & $\begin{array}{l}35 \\
19.7 \text { to } 53.5\end{array}$ & $\begin{array}{l}27 \\
11.6 \text { to } 47.8\end{array}$ & $\begin{array}{l}25 \\
9.8 \text { to } 46.7\end{array}$ & $\begin{array}{l}27 \\
10.7 \text { to } 50.2\end{array}$ & $\begin{array}{l}52 \\
29.8 \text { to } 74.3\end{array}$ & $\begin{array}{l}81 \\
58.1 \text { to } 94.6\end{array}$ & $\begin{array}{l}50 \\
26.0 \text { to } 74.0\end{array}$ & $\begin{array}{l}50 \\
24.7 \text { to } 75.3\end{array}$ \\
\hline $\begin{array}{l}\text { DCR, } \\
95 \% \mathrm{Cl}\end{array}$ & $\begin{array}{l}55 \\
41.5 \text { to } 68.7\end{array}$ & $\begin{array}{l}38 \\
22.2 \text { to } 56.4\end{array}$ & $\begin{array}{l}35 \\
17.2 \text { to } 55.7\end{array}$ & $\begin{array}{l}29 \\
12.6 \text { to } 51.1\end{array}$ & $\begin{array}{l}41 \\
20.7 \text { to } 63.6\end{array}$ & $\begin{array}{l}52 \\
29.8 \text { to } 74.3\end{array}$ & $\begin{array}{l}86 \\
63.7 \text { to } 97.0\end{array}$ & $\begin{array}{l}56 \\
30.8 \text { to } 78.5\end{array}$ & $\begin{array}{l}50 \\
24.7 \text { to } 75.3\end{array}$ \\
\hline
\end{tabular}

${ }^{*}$ Cancers with centrally confirmed MSI-H/dMMR.

†Confirmed responses per RECIST V.1.1.

¥CBR was defined as the rate of patients who achieved CR, PR, or SD with a duration $\geq 16$ weeks.

BOR, best overall response; CBR, clinical benefit rate; CR, complete response; DCR, disease control rate; dMMR, deficient mismatch repair; ESCC, esophageal squamous cell carcinoma; GC, gastric cancer; HCC, hepatocellular carcinoma; MSI-H, microsatellite instability-high; NE, not estimable; NPC, nasopharyngeal carcinoma; NSCLC, non-small cell lung cancer; ORR, objective response rate; PD, progressive disease; PR, partial response; RCC, renal cell carcinoma; RECIST, Response Evaluation In Solid Tumors; SD, stable disease; UC, urothelial bladder cancer.

non-keratinizing subtype is predominantly associated with Epstein-Barr virus (EBV) infection and is the most common subtype of NPC, representing $>95 \%$ of cases in areas where NPC is more prevalent such as Southern China; the keratinizing subtype accounts for less than $20 \%$ of worldwide cases of NPG and is fairly rare in Southern China. ${ }^{31}$ PD-L1 is expressed in approximately $90 \%$ of EBV-associated NPCs, ${ }^{32}$ which makes inhibitors of the PD-1/PD-L1 pathway attractive treatment options for EBV-associated NPC. In the current study, the ORR in patients with NPC treated with tislelizumab was $43 \%$ and the median DoR was 8.3 months (95\% CI 3.9 to not estimable), with a median follow-up of 4.8 months (range 2.1-11.1); median PFS was 10.4 months (95\% CI 4.2, 10.5) and the median OS had not yet been reached. A recent multinational study showed that nivolumab resulted in an ORR of $20.5 \%$ in 44 patients with NPC; median PFS was 2.8 months and median OS was 17.1 months. ${ }^{33}$ In a different study, treatment of 27 patients with PD-L1positive NPG with pembrolizumab resulted in seven PRs for an ORR of 25.9\%; median OS was 16.5 months and median PFS was 6.5 months. ${ }^{34}$ Differences between these earlier studies and the current data could possibly be explained by differences in patient populations, as the current study exclusively enrolled patients with non-keratinizing NPC. However, the preliminary safety profile and antitumor activity of tislelizumab warrant its continued development, and a phase 3 study in patients with NPC (NCT03924986) has been initiated.

While cytotoxic chemotherapy has been historically used for the treatment of patients with advanced NSCLC who do not have a targetable molecular driver mutation, survival benefit was limited and these treatments often resulted in significant toxicity. ${ }^{35}$ Treatment with immunotherapeutic agents targeting the PD-1/PD-L1 checkpoint inhibitor pathway has drastically changed the treatment paradigms for these patients. Pembrolizumab is approved for use in patients with NSCLC and has demonstrated superiority to platinum-containing doublet chemotherapy in patients expressing high levels of PD-L1; two additional immune checkpoint inhibitors (atezolizumab and nivolumab) have been approved for second-line use in patients with NSCLC. ${ }^{35}$ In the Keynote-001 study, the ORR of pembrolizumab was $19.4 \%$ (95\% CI 16.0, 23.2) in 495 patients with NSCLC. In the phase 3 CheckMate-078 study, which was predominantly comprised of patients with previously treated NSCLC from China, the ORR of patients treated with nivolumab was $17 \%$ compared with $4 \%$ with docetaxel. ${ }^{27}$ Notably, our report demonstrates a comparable ORR (18\%) in heavily treated Chinese patients with NSCLC. Based on the data from this study and other additional clinical trials, three phase 3 studies of tislelizumab as treatment for NSCLC have been initiated (NCT03358875, NCT03594747, and NCT03663205). 
A

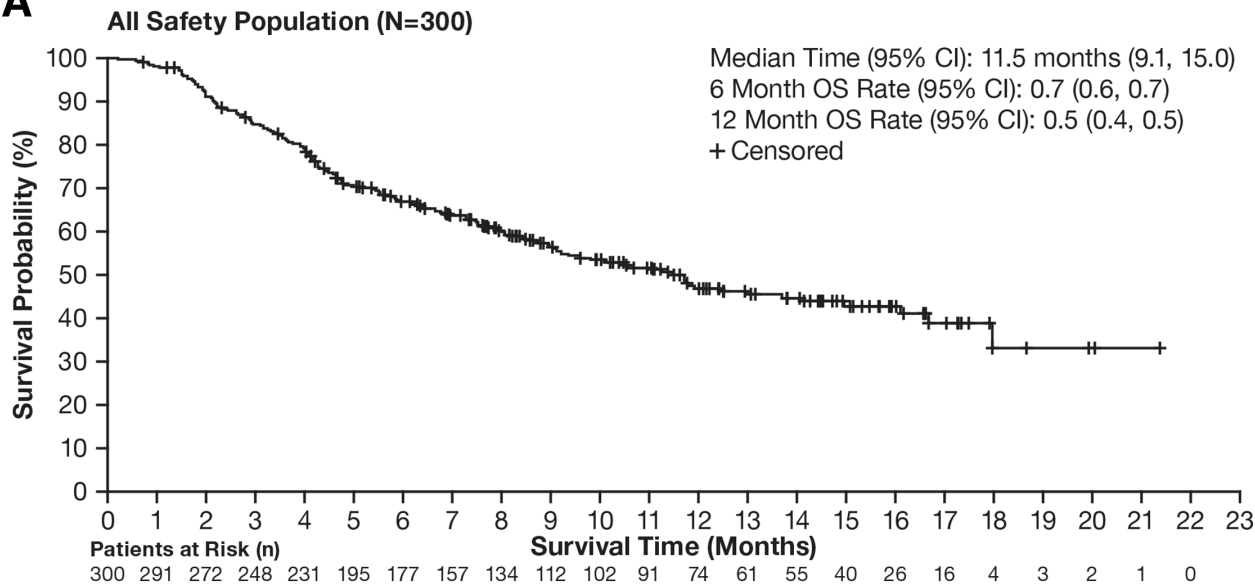

B

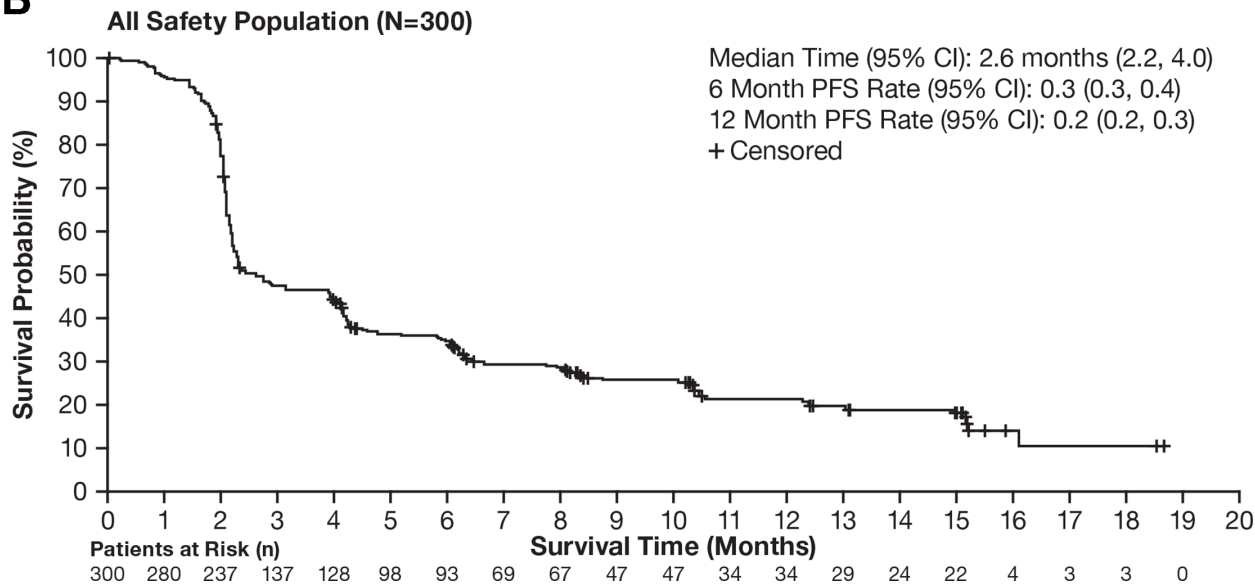

Figure 1 (A) Overall survival (safety analysis set). (B) Progression-free survival (safety analysis set). OS, overall survival; PFS, progression-free survival.

GC is the second most common cancer in China. ${ }^{36}$ Currently, most patients with advanced GC are treated with chemotherapy, but the prognosis and OS rates remain low. ${ }^{37}$ PD-L1 overexpression has been detected in tumors from patients with gastric or gastroesophageal junction cancer (GC/GEJC) ${ }^{38}$ making antibody therapies that target PD-1/PD-L1 potential treatment options for patients with GC/GEJC. The phase 3 ATTRACTION-2 trial has demonstrated that nivolumab can improve OS with manageable AEs and durable responses in patients with unresectable advanced or recurrent GC/GEJC that has progressed after chemotherapy in Asian patients, with an ORR of $11 \%$ and median OS of 5.3 months. ${ }^{39}$ These results were consistent with a large phase 2 trial in which 259 patients with previously treated GC or GEJC treated with pembrolizumab monotherapy had an ORR of $12 \%$ and a median OS of 5.6 months. ${ }^{40}$ With a median OS of 4.7 months and an ORR of $17 \%$, the current study demonstrated slightly higher antitumor activity compared with that of nivolumab and pembrolizumab. Etiological differences between Western and Asian populations, different baseline disease profiles, and limited sample size may have contributed to the differences in ORRs observed between the current study and those of other anti-PD-1 antibody therapies. A phase 3 study (NCT03777657) has been initiated to further assess the efficacy and safety of tislelizumab in combination with chemotherapy as firstline treatment of GC.

Based on histological classification, the two largest subtypes of esophageal cancer are squamous cell carcinoma and adenocarcinoma. The majority of esophageal cancers in China are histologically classified as squamous cell carcinoma, while in North America, Western Europe, and Australia, adenocarcinoma has become the most common histological subtype of esophageal cancer. ${ }^{28} 30$ Although the underlying mechanisms remain unclear, emerging data suggest anti-PD-1 monotherapy may have increased antitumor activity in ESCC when compared with esophageal adenocarcinoma. ${ }^{41}$ The US FDA approved pembrolizumab as monotherapy for second-line ESCC in patients whose tumors express PD-L1 (combined positive score $(\mathrm{CPS}) \geq 10$ ) based on the data from two clinical trials,${ }^{42}$ including the phase 3 KEYNOTE-181 trial. In the KEYNOTE-181 study, the median OS for patients with CPS $\geq 10$ ESCC was 10.3 months compared with 6.7 months for those receiving standard-of-care chemotherapy; median OS for patients with CPS $\geq 10$ esophageal adenocarcinoma was 6.3 months for those receiving 


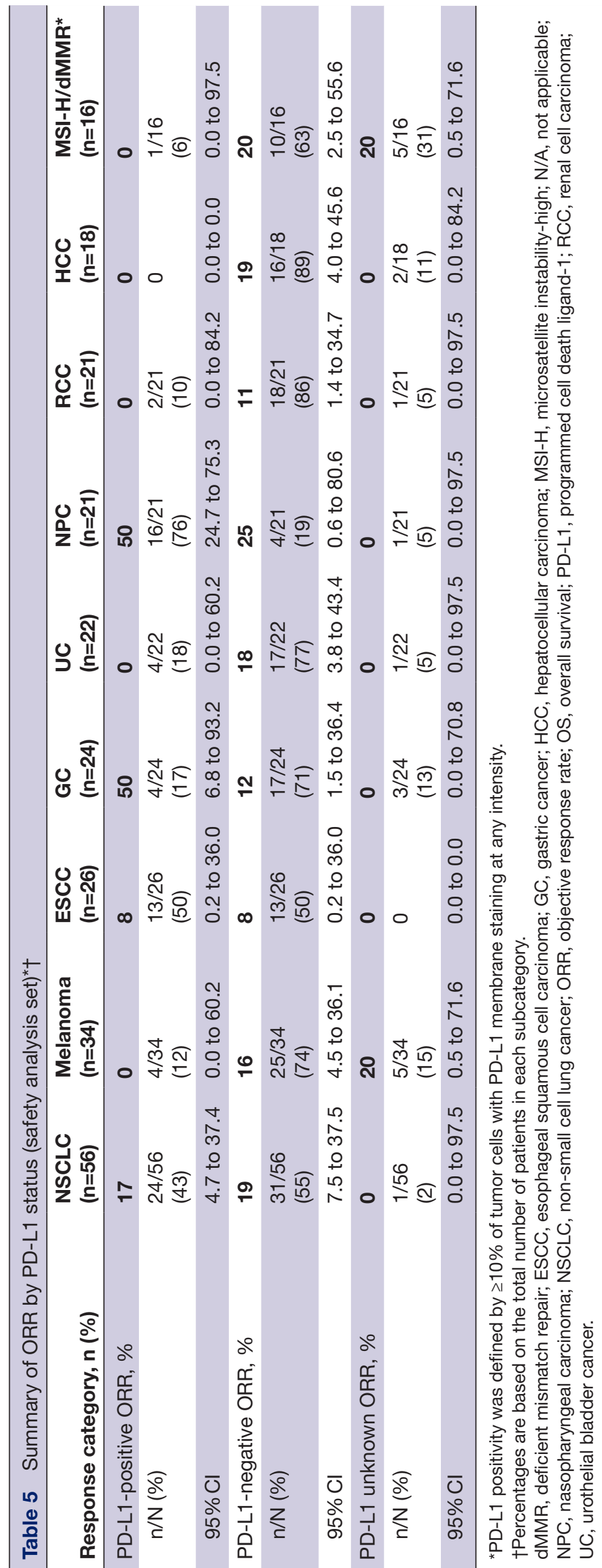



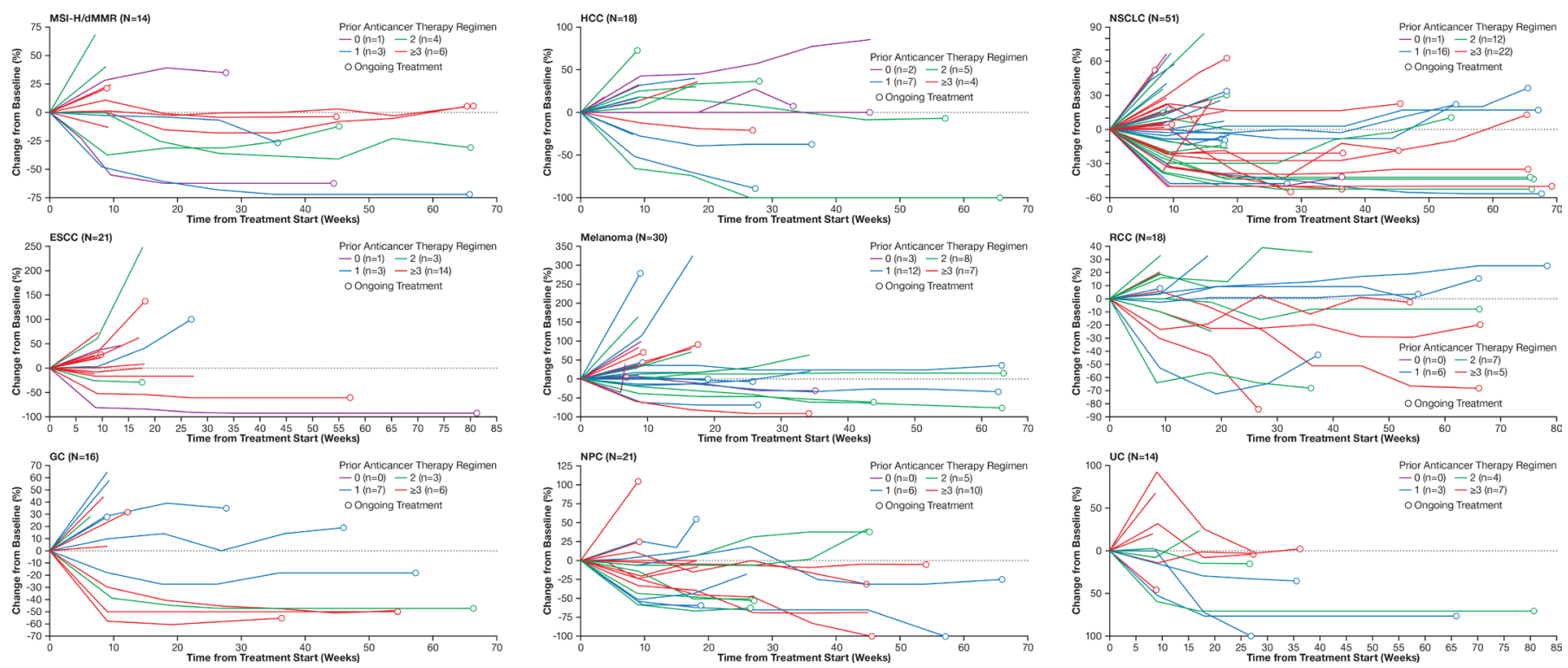

Figure 2 Change of total sum of target lesion diameters from baseline abbreviations: dMMR, deficient mismatch repair; ESCC, esophageal squamous cell carcinoma; GC, gastric cancer; HCC, hepatocellular carcinoma; MSI-H; microsatellite instabilityhigh; NPC, nasopharyngeal carcinoma; NSCLC, non-small cell lung cancer; RCC, renal cell carcinoma; UC, urothelial bladder cancer.

pembrolizumab versus 6.9 months for those receiving standard-of-care chemotherapy. ${ }^{43}$ These data suggest that the OS benefit from pembrolizumab in patients with esophageal cancer is driven by the ESCC subgroup. At 8\%, the ORR of patients with ESCC from the current study is lower than previous reports of other anti-PD-1 antibody therapies (the ORR in patients with ESCC was $16.7 \%$ in the KEYNOTE-181 trial ${ }^{44}$ ), possibly because of limited sample size or because patients in the current study were more heavily pretreated $(77 \%$ of patients with ESCC had $\geq 2$ lines of prior systemic therapy; online supplementary table S1). However, with a DCR of $29 \%$, the current study is comparable with previous reports of pembrolizumab in heavily pretreated patients with advanced, metastatic esophageal cancer $(31 \%) .{ }^{45}$ Three phase 3 trials have been initiated to evaluate the efficacy and safety of tislelizumab alone and in combination with chemotherapy/chemoradiotherapy in patients with ESCC (NCT03783442, NCT03430843, and NCT03957590).

Based on the anatomical location and the degree of sun exposure, melanoma is classified into four major subtypes. Acral melanoma is rare among the white population but has a higher incidence in Asians. When compared with other melanoma subtypes, acral melanoma may represent a more biologically aggressive subtype with a worse prognosis. ${ }^{46}$ We observed an ORR of $15 \%$ in the current study, which is similar to data from KEYNOTE-151, a phase 1B study of second-line pembrolizumab for Chinese patients with advanced melanoma (ORR 17\%). ${ }^{47}$ Furthermore, patients with melanoma had a long duration of treatment (17.9 months) and durable responses (DoR, not estimable) in the current study, and tislelizumab demonstrated preliminary antitumor activity in Chinese patients with pretreated melanoma.
One characteristic of MSI-H/dMMR tumors is that they have a high tumor mutational burden, which is believed to be one of the reasons these tumors respond well to PD-1blocking antibodies. ${ }^{48}$ In the KEYNOTE-164 study, 63 patients with MSI-H metastatic colorectal cancer (mCRC) treated with pembrolizumab had an ORR of $32 \% .{ }^{49}$ Additionally, in the phase 2 CheckMate-142 trial, which examined responses to nivolumab in 74 patients with MSI-H/ dMMR mCRC, 23 patients $(31 \%)$ had an investigatorassessed objective response. ${ }^{50}$ With an ORR of $19 \%$, the current study is the first to report clinical responses of an anti-PD-1-antibody treatment in Chinese patients with MSI-H/dMMR solid tumors, and it is therefore unclear if patient population contributed to differential responses between these studies. The limited sample size of this cohort may also have led to the differences in response compared with previous studies. Further investigation is needed before a correlation between a patient's race and their response to PD-1 inhibitor therapy can be made. A phase 2 study (NCT03736889) to further investigate the efficacy of tislelizumab in tumors with MSI-H/dMMR is ongoing.

\section{CONCLUSIONS}

Tislelizumab was generally well tolerated and no unexpected safety issues were seen in this study. Preliminary antitumor activity was observed in patients with multiple solid tumors regardless of PD-L1 expression. Overall, the antitumor activity of tislelizumab was consistent with other PD-1 inhibitors and the results of this study support further investigation of tislelizumab in specific solid tumors. 


\section{Author affiliations}

${ }^{1}$ Key Laboratory of Carcinogenesis and Translational Research (Ministry of Education/Beijing), Peking University Cancer Hospital and Institute, Beijing, China ${ }^{2}$ Department of Medical Oncology, Harbin Medical University Cancer Hospital, Harbin, China

${ }^{3}$ Department of Medical Oncology, Sir Run Run Shaw Hospital, Zhejiang University, School of Medicine, Hangzhou, China

${ }^{4}$ The Cancer Institute Key Laboratory of Cancer Prevention and Intervention, Chinese National Ministry of Education, The Second Affiliated Hospital Zhejiang University School of Medicine, Zhejiang, China

${ }^{5}$ Department of Medical Oncology, Zhongshan Hospital Fudan University, Shanghai, China

${ }^{6}$ Guangdong Lung Cancer Institute, Guangdong Provincial People's Hospital and Guangdong Academy of Medical Sciences, Guangzhou, China

${ }^{7}$ Department of Medical Oncology, Jiangsu Province People's Hospital, Hangzhou, China

${ }^{8}$ Guangdong Provincial Key Laboratory of Malignant Tumor Epigenetics and Gene Regulation, Sun Yat-sen Memorial Hospital, Sun Yat-sen University, Zhuhai, China ${ }^{9}$ Department of Radiation Oncology, The Fifth Affiliated Hospital, Sun Yat-Sen University, Zhuhai, China

${ }^{10}$ Cancer Institute and Hospital, Chinese Academy of Medical Sciences, Beijing, China

${ }^{11}$ Department of Urology, Fudan University Shanghai Cancer, Shanghai, China

${ }^{12}$ Department of Urology, The First Affiliated Hospital of Nanchang University,

Nanchang, China

${ }^{13}$ BeiGene (Beijing) Co., Ltd, Beijing, China

Correction notice This article has been corrected since it was published Online First. The author name Zuobai Wang was incorrectly spelt as Zoubai Wang.

Acknowledgements The authors wish to acknowledge the investigative center study staff, the study patients, and their families. Financial support for the development of this manuscript, including medical writing and editorial assistance under the authors' guidance, provided by Stephan Lindsey, $\mathrm{PhD}$, and Elizabeth Hermans, PhD, of OPEN Health Medical Communications (Chicago, Illinois, USA), was provided by BeiGene.

Contributors The study protocol was developed by BeiGene in collaboration with the study investigators. BeiGene was also involved in data collection, analysis, and interpretation of results. Statistical analyses were performed by statisticians at BeiGene. All authors were in agreement regarding the submission of this manuscript and endorse the completeness and accuracy of the data. The corresponding author had full access to all of the study data and was responsible for the decision to submit the manuscript for publication.

Funding The study protocol was developed by BeiGene in collaboration with the study investigators. BeiGene was also involved in data collection, analysis, and interpretation of results. Statistical analyses were performed by statisticians at BeiGene.

Competing interests LS, JG, QZ, HP, YY, YB, TL, QZ, JZ, YS, XH, SW, JW, AZ, DY, TS, and Y-LW have nothing to declare. YG, SY, ZW, and JL are employees of BeiGene.

Patient consent for publication Not required.

Ethics approval This study was performed in accordance with the ethical principles of the Declaration of Helsinki, Good Clinical Practice guidelines, and the principles of informed consent. Written informed consent was obtained from each patient prior to screening. The protocol was approved by an independent ethics committee prior to initiation.

Provenance and peer review Not commissioned; externally peer reviewed.

Data availability statement Data are available on reasonable request. Upon request, and subject to certain criteria, conditions, and exceptions, BeiGene will provide access to individual deidentified participant data from BeiGene-sponsored global interventional clinical studies conducted for medicines (1) for indications that have been approved or (2) in programs that have been terminated. BeiGene will also consider requests for the protocol, data dictionary, and statistical analysis plan. Data requests may be submitted to medicalinformation@beigene. com.

Open access This is an open access article distributed in accordance with the Creative Commons Attribution Non Commercial (CC BY-NC 4.0) license, which permits others to distribute, remix, adapt, build upon this work non-commercially, and license their derivative works on different terms, provided the original work is properly cited, appropriate credit is given, any changes made indicated, and the use is non-commercial. See http://creativecommons.org/licenses/by-nc/4.0/.

\section{ORCID iDs}

Jie Wang http://orcid.org/0000-0002-5602-0487

Yi-Long Wu http://orcid.org/0000-0002-3611-0258

\section{REFERENCES}

1 GBD 2016 Causes of Death Collaborators. Global, regional, and national age-sex specific mortality for 264 causes of death, 19802016: a systematic analysis for the global burden of disease study 2016. Lancet 2017;390:1151-210.

2 Bray F, Ferlay J, Soerjomataram I, et al. Global cancer statistics 2018: GLOBOCAN estimates of incidence and mortality worldwide for 36 cancers in 185 countries. CA Cancer J Clin 2018;68:394-424.

3 Pilleron S, Sarfati D, Janssen-Heijnen M, et al. Global cancer incidence in older adults, 2012 and 2035: a population-based study. Int J Cancer 2019;144:49-58.

4 Zeng H, Chen W, Zheng R, et al. Changing cancer survival in China during 2003-15: a pooled analysis of 17 population-based cancer registries. Lancet Glob Health 2018;6:e555-67.

5 McDermott DF, Atkins MB. PD-1 as a potential target in cancer therapy. Cancer Med 2013;2:n/a-73.

6 Azuma K, Ota K, Kawahara A, et al. Association of PD-L1 overexpression with activating EGFR mutations in surgically resected nonsmall-cell lung cancer. Ann Oncol 2014;25:1935-40.

7 Okita R, Maeda A, Shimizu K, et al. PD-L1 overexpression is partially regulated by EGFR/HER2 signaling and associated with poor prognosis in patients with non-small-cell lung cancer. Cancer Immunol Immunother 2017;66:865-76.

8 Massi D, Brusa D, Merelli B, et al. The status of PD-L1 and tumorinfiltrating immune cells predict resistance and poor prognosis in BRAFi-treated melanoma patients harboring mutant BRAFV600. Ann Oncol 2015;26:1980-7.

9 Wang H, Yao H, Li C, et al. PD-L2 expression in colorectal cancer: independent prognostic effect and targetability by deglycosylation. Oncoimmunology 2017;6:e1327494.

10 Wang X, Teng F, Kong L, et al. PD-L1 expression in human cancers and its association with clinical outcomes. Onco Targets Ther 2016;9:5023-39.

11 Wu P, Wu D, Li L, et al. PD-L1 and survival in solid tumors: a metaanalysis. PLoS One 2015;10:e0131403.

12 Fang W, Zhang J, Hong S, et al. EBV-driven LMP1 and IFN- $\gamma$ up-regulate PD-L1 in nasopharyngeal carcinoma: implications for oncotargeted therapy. Oncotarget 2014;5:12189-202.

13 Mahoney KM, Freeman GJ, McDermott DF. The next immunecheckpoint inhibitors: PD-1/PD-L1 blockade in melanoma. Clin Ther 2015;37:764-82.

14 Wang B, Qin L, Ren M, et al. Effects of combination of anti-CTLA-4 and anti-PD-1 on gastric cancer cells proliferation, apoptosis and metastasis. Cell Physiol Biochem 2018;49:260-70.

15 Topalian SL, Hodi FS, Brahmer JR, et al. Safety, activity, and immune correlates of anti-PD-1 antibody in cancer. N Engl J Med 2012;366:2443-54

16 Zhang T, Song X, Xu L, et al. The binding of an anti-PD-1 antibody to FcyRI has a profound impact on its biological functions. Cancer Immunol Immunother 2018:67:1079-90.

17 Dahan R, Sega E, Engelhardt $J$, et al. FcyRs modulate the anti-tumor activity of antibodies targeting the PD-1/PD-L1 axis. Cancer Cell 2015;28:285-95.

18 Feng $\mathrm{Y}$, Hong $\mathrm{Y}$, Sun $\mathrm{H}$, et al. The molecular binding mechanism of tislelizumab, an investigational anti-PD-1 antibody, is differentiated from pembrolizumab and nivolumab. in: proceedings of the 110th annual meeting of the American association for cancer research. Atlanta, GA: American Association of Cancer Research, 2019.

19 Desai J, Markman B, Sandhu SK, et al. A phase I dose-escalation study of BGB-A317, an anti-programmed death-1 (PD-1) mAb in patients with advanced solid tumors. $J$ Immunother Cancer 2016;4:P154.

20 Deva S, Lee J-S, Lin C-C, et al. A phase la/lb trial of tislelizumab, an anti-PD-1 antibody (ab), in patients (PTS) with advanced solid tumors. Annals of Oncology 2018;29:×24-5.

21 Eisenhauer EA, Therasse P, Bogaerts J, et al. New response evaluation criteria in solid tumours: revised RECIST guideline (version 1.1). Eur J Cancer 2009;45:228-47.

22 Scorer P, Scott M, Lawson N, et al. Consistency of tumor and immune cell programmed cell death ligand-1 expression within and 
between tumor blocks using the VENTANA SP263 assay. Diagn Pathol 2018;13:47.

23 Sznol M, Ferrucci PF, Hogg D, et al. Pooled analysis safety profile of nivolumab and ipilimumab combination therapy in patients with advanced melanoma. J Clin Oncol 2017;35:3815-22.

24 Desai J, Millward M, Chao Y, et al. Preliminary results from subsets of patients (PTS) with advanced gastric cancer (GC) and esophageal carcinoma (EC) in a dose-escalation/expansion study of BGB-A317, an anti-PD-1 monoclonal antibody (mAb). Ann Oncol 2017:28:122-41.

25 Horvath L, Desai J, Sandhu S, et al. Preliminary results from a subset of patients (PTS) with advanced head and neck squamous carcinoma (HNSCC) in a dose-escalation and dose-expansion study of BGB-A317, an anti-PD-1 monoclonal antibody (mAb). Ann Oncol 2017:28:388P

26 Song $\mathrm{Y}, \mathrm{Gao} \mathrm{Q}$, Zhang $\mathrm{H}$, et al. Treatment of relapsed or refractory classical Hodgkin lymphoma with the anti-PD-1, tislelizumab: results of a phase 2 , single-arm, multicenter study. Leukemia 2020;34:533-42.

27 Wu Y-L, Lu S, Cheng Y, et al. Nivolumab versus docetaxel in a predominantly Chinese patient population with previously treated advanced NSCLC: CheckMate 078 randomized phase III clinical trial. $J$ Thorac Oncol 2019:14:867-75.

28 Abbas G, Krasna M. Overview of esophageal cancer. Ann Cardiothorac Surg 2017;6:131-6.

29 Feng R-M, Zong Y-N, Cao S-M, et al. Current cancer situation in China: good or bad news from the 2018 global cancer statistics? Cancer Commun 2019;39:22.

30 Feng Y, Wu N. Management guidelines for esophageal carcinoma 2018: Chinese standards in clinical practice. Chin J Cancer Res 2019;31:421-2.

31 Chen Y-P, Chan ATC, Le Q-T, et al. Nasopharyngeal carcinoma. Lancet 2019;394:64-80.

32 Chen BJ, Chapuy B, Ouyang J, et al. PD-L1 expression is characteristic of a subset of aggressive B-cell lymphomas and virusassociated malignancies. Clin Cancer Res 2013;19:3462-73.

33 Ma BBY, Lim W-T, Goh B-C, et al. Antitumor activity of nivolumab in recurrent and metastatic nasopharyngeal carcinoma: an international, multicenter study of the Mayo clinic phase 2 Consortium (NCl-9742). $J$ Clin Oncol 2018;36:1412-8.

34 Hsu C, Lee S-H, Ejadi S, et al. Safety and antitumor activity of pembrolizumab in patients with programmed death-ligand 1-positive nasopharyngeal carcinoma: results of the KEYNOTE-028 study. $J$ Clin Oncol 2017;35:4050-6.

35 Pabani A, Butts CA. Current landscape of immunotherapy for the treatment of metastatic non-small-cell lung cancer. Curr Oncol 2018;25:94-102.

36 Chen W, Zheng R, Baade PD, et al. Cancer statistics in China, 2015. CA Cancer J Clin 2016;66:115-32.
37 Jou E, Rajdev L. Current and emerging therapies in unresectable and recurrent gastric cancer. World J Gastroenterol 2016;22:4812-23.

38 Tran PN, Sarkissian S, Chao J, et al. PD-1 and PD-L1 as emerging therapeutic targets in gastric cancer: current evidence. Gastrointest Cancer 2017;7:1-11.

39 Kang Y-K, Boku N, Satoh T, et al. Nivolumab in patients with advanced gastric or gastro-oesophageal junction cancer refractory to, or intolerant of, at least two previous chemotherapy regimens (ONO-4538-12, ATTRACTION-2): a randomised, double-blind, placebo-controlled, phase 3 trial. Lancet 2017;390:2461-71.

40 Fuchs CS, Doi T, Jang RW, et al. Safety and efficacy of pembrolizumab monotherapy in patients with previously treated advanced gastric and gastroesophageal junction cancer: phase 2 clinical KEYNOTE-059 trial. JAMA Oncol 2018;4:e180013.

41 Kelly RJ. The emerging role of immunotherapy for esophageal cancer. Curr Opin Gastroenterol 2019;39:337-43.

42 US Food and Drug Adminstration. FDA approves pembrolizumab for advanced esophageal squamous cell cancer, 2019. Available: https://www.fda.gov/drugs/resources-information-approved-drugs/ fda-approves-pembrolizumab-advanced-esophageal-squamouscell-cancer [Accessed 5 Sep 2019].

43 Shah MA, Adenis A, Enzinger PC, et al. Pembrolizumab versus chemotherapy as second-line therapy for advanced esophageal cancer: phase 3 KEYNOTE-181 study. JCO 2019;37:4010.

44 Kojima T, Muro K, Francois E, et al. Pembrolizumab versus chemotherapy as second-line therapy for advanced esophageal cancer: phase III KEYNOTE-181 study. JCO 2019;37:2.

45 Shah MA, Kojima T, Hochhauser D, et al. Efficacy and safety of pembrolizumab for heavily pretreated patients with advanced, metastatic adenocarcinoma or squamous cell carcinoma of the esophagus: the phase 2 KEYNOTE-180 study. JAMA Oncol 2019;5:546-50.

46 Dai B, Cai X, Kong Y-Y, et al. Analysis of kit expression and gene mutation in human acral melanoma: with a comparison between primary tumors and corresponding metastases/recurrences. Hum Pathol 2013;44:1472-8.

47 Si L, Zhang X, Shu Y, et al. A phase lb study of pembrolizumab as second-line therapy for Chinese patients with advanced or metastatic melanoma (KEYNOTE-151). Trans/ Oncol 2019:12:828-35.

48 Kok M, Chalabi M, Haanen J. How I treat MSI cancers with advanced disease. ESMO Open 2019:4:e000511.

49 DT L, Kavan P, Kim TW, et al. KEYNOTE-164: pembrolizumab for patients with advanced microsatellite instability high (MSI-H) colorectal cancer. J Clin Oncol 2018;36:3514.

50 Overman MJ, McDermott R, Leach JL, et al. Nivolumab in patients with metastatic DNA mismatch repair-deficient or microsatellite instability-high colorectal cancer (CheckMate 142): an open-label, multicentre, phase 2 study. Lancet Oncol 2017;18:1182-91. 
Correction: Tislelizumab in Chinese patients with advanced solid tumors: an open-label, non-comparative, phase $1 / 2$ study

Shen L, Guo J, Zhang Q, et al. Tislelizumab in Chinese patients with advanced solid tumors: an open-label, non-comparative, phase 1/2 study. J immunother Cancer 2020;8:e000437. doi: 10.1136/jitc-2019-000437

Since the online publication of this article, the authors have noticed that the author name Zuobai Wang was incorrectly spelt as Zoubai Wang. This has been corrected.

Open access This is an open access article distributed in accordance with the Creative Commons Attribution Non Commercial (CC BY-NC 4.0) license, which permits others to distribute, remix, adapt, build upon this work non-commercially, and license their derivative works on different terms, provided the original work is properly cited, appropriate credit is given, any changes made indicated, and the use is non-commercial. See: http://creativecommons.org/licenses/by-nc/4.0/.

C Author(s) (or their employer(s)) 2020. Re-use permitted under CC BY-NC. No commercial re-use. See rights and permissions. Published by BMJ.

J Immunother Cancer 2020;8:e000437corr1 . doi:10.1136/jitc-2019-000437corr1

A) Check for updates 